\title{
General Switch-and-Stay Combing for Space Diversity over Rayleigh Fading Channels
}

\author{
Yawgeng A. Chau' ${ }^{1}$ and Yao-Hua Chen ${ }^{2}$ \\ ${ }^{1}$ Department of Communications Engineering, Yuan Ze University, Chungli 320, Taiwan \\ ${ }^{2}$ Information and Communications Research Laboratories, Industrial Technology Research Institute, Hsinchu 310, Taiwan
}

Correspondence should be addressed to Yawgeng A. Chau, eeyaw@saturn.yzu.edu.tw

Received 26 December 2011; Accepted 17 February 2012

Academic Editor: Tat Yeo

Copyright ( 2012 Y. A. Chau and Y.-H. Chen. This is an open access article distributed under the Creative Commons Attribution License, which permits unrestricted use, distribution, and reproduction in any medium, provided the original work is properly cited.

\begin{abstract}
Three multibranch switch-and-stay combining (MSSC) schemes are analyzed for Rayleigh fading channels, where different decision statistics for antenna switching (i.e., switch statistic) are used. Let $a$ and $r$ denote the fading factor and the received baseband signal of a diversity branch, respectively. In contrast to the traditional MSSC that uses the faded signal-to-noise ratio (SNR) of diversity branches as the corresponding switch statistic, to enhance the receiver performance, $|r|$, $|a r|$, and a new linear combination of a and $|r|$ are used as switch statistics of the three MSSC schemes, respectively. For performance evaluation, the bit error rate (BER) of BPSK is derived for the three MSSC schemes over both independent-and-identical distributed (i.i.d.) and independent-and-nonidentical distributed (i.n.d.) Rayleigh fading channels. To pursue optimal performance, the locally optimal switch threshold (ST) of each MSSC scheme is obtained for general i.n.d. fading channels. In addition, the locally optimal ST becomes the globally optimal ST for i.i.d. channels. Numerical results based on the analysis and simulations are presented. In contrast to the MSSC over i.i.d. fading channels, we will show that the performance of MSSC schemes can be improved by increasing the number of branches, if $i . n$.d. channels are considered.
\end{abstract}

\section{Introduction}

In a wireless system, diversity combining provides an effective way to overcome multipath fading and enhance receiver performance [1-6]. The well-known maximalratio-combining and equal-gain-combining schemes require numerous arithmetic operations such as adders and/or multipliers for the implementation of signal combination. On the other hand, the conventional selection combining (SC) scheme needs simultaneous and continuous monitoring and estimation of all branch SNRs. The arithmetic operations and continuous signal-to-noise ratio (SNR) estimations are time- and power-consuming, which may be impractical for some wireless communication systems [5, 7]. To further reduce the implementation complexity of a diversity system, the switch-and-stay combining (SSC) scheme has been considered [8-12]. With an SSC system, the receiver only needs to monitor and estimate the channel state of the single branch currently in use. Thus, the switch-based SSC is valuable for a mobile terminal that has a limited processing capability.

For traditional switch-based diversity schemes, the faded SNR of diversity branches is usually used as the decision statistics for antenna switching (i.e., switch statistic) in a threshold testing procedure and can be categorized as the SNR-based combining scheme. In [9], the SNR-based dualbranch SSC scheme was proposed, and the bit error rate (BER) of noncoherent FSK (NCFSK) was derived. In [10], a simplified dual-branch SNR-based SSC scheme was analyzed for the NCFSK on independent and correlated Nakagami fading channels. In [11], the dual-branch SNR-based SSC scheme on general fading channels was analyzed, and the optimal switch threshold (ST) was studied. In [12], the SSC scheme addressed in [9] and [10] was extended to the case of independent multibranch channels. In [13], the dual-branch SSC scheme in correlated Rayleigh and Rician fading channels was analyzed. In [14-16], the SSC scheme was applied to wireless communication systems that involve 
relays. In the previous works on the SSC scheme [7-16], only the faded SNR is considered as the switch statistic in the procedure of threshold testing, where an identical ST is used. Once the SNR of the used branch is lower than a preset threshold, the receiver switches to the next branch for signal reception. In a multibranch SSC (MSSC) system, the switchto branch of the last branch will be the first one. Although the SSC is less complex than the SC, the SNR-based SSC suffers from a considerable performance loss compared to its counterpart of SC.

Recently, other statistics rather than the SNR have been considered for the SC and associated branch selections. Let $a$ and $r$ be the fading factor and the received baseband signal of a diversity branch, respectively. In the SC scheme proposed in [17], the largest $|r|$ at the receiver site is selected for signal detection on i.i.d. Rayleigh fading channels. In [18], the SC by choosing the largest $|a r|$ was analyzed for BPSK signaling also on i.i.d. Rayleigh fading channels.

In this paper, we want to explore possible performance improvement compared to traditional SNR-based SSC and SC schemes, when $|r|$ or $|a r|$ are used as the switch statistic of MSSC schemes. In addition, as an alternative choice for generating a statistic with lower complexity than $|a r|$, a linear combination of $a$ and $|r|$ is considered as the new switch statistic. Specifically, in contrast to the traditional SNR-based SSC and SC schemes, $|r|,|a r|$, and the linear combination of $a$ and $|r|$ are used as three different new switch statistics of MSSC schemes, respectively, where the performance of BPSK on i.i.d. and i.n.d. Rayleigh fading channels is evaluated. Notice that a variable multiplier is required to generate the statistic $|a r|$ for each branch, while only adders are used for the linear combination of $a$ and $|r|$. It is well know from circuit design that an adder is much less complex than a variable multiplier. Thus, among the three MSSC schemes, the MSSC based on $|a r|$ has the highest implementation complexity.

To optimize the performance of each MSSC scheme, an optimal or locally optimal ST that minimizes the BER of BPSK is explored. For the general i.n.d. fading channels, the globally optimal ST is difficult to obtain, and thus the locally optimal ST is developed for each diversity branch. A nice result, is that, for the i.i.d. fading channels with an identical ST, the locally optimal ST is also globally optimal.

In published literature $[10,12]$, it has been shown that the performance of MSSC schemes on traditional i.i.d. fading channels cannot be enhanced by adding more branches for diversity combining, and the diversity gain remains the same as the one of dual-branch case. In this paper, we will also show that if i.n.d. fading channels are considered, the performance of MSSC schemes can be further improved with more than two branches for combining.

The remainder of the paper is organized as follows. In Section 2, the MSSC models with different switch statistics for BPSK on independent Rayleigh fading channels are presented. In Section 3, the corresponding BER of BPSK is derived for the three MSSC schemes on i.i.d. and i.n.d. fading channels, respectively. In Section 4, the optimal ST of the three MSSC schemes is derived for performance optimization, where an optimal ST in closed-form is obtained for the $|a r|$-based MSSC. In Section 5, numerical results based on the analysis and simulations are presented. Conclusions are drawn in Section 6.

\section{Modeling of General MSSC}

2.1. Signal and Channel Model. For $L$-branch diversity combining, let $E_{b}$ be the received energy, and $a_{l}$ denote the fading factor of branch $l$ for $l=1,2, \ldots, L$. For BPSK signaling, the received signal after phase compensation for branch $l$ can be represented by [14]

$$
r_{l}=a_{l} s_{m}+n_{l}, \quad l=1,2, \ldots, L
$$

where $m=0,1, s_{0}=-\sqrt{E_{b}}, s_{1}=\sqrt{E_{b}}$, and $n_{l}$ is the additive complex Gaussian noise with zero mean and the variance $N_{0} / 2$. The faded SNR of branch $l$ is given by $\gamma_{l}=a_{l}^{2} E_{b} / N_{0}$.

For independent Rayleigh fading channels, the probability density function (PDF) of $\gamma_{l}$ is given by

$$
f_{\gamma_{l}}(x)=\frac{1}{\bar{\gamma}_{l}} e^{-\left(x / \bar{\gamma}_{l}\right)}, \quad x \geq 0,
$$

where $\bar{\gamma}_{l}=E\left[\gamma_{l}\right]$. The corresponding cumulative distribution function (CDF) of $y_{l}$ is

$$
F_{\gamma_{l}}(x)=1-e^{-\left(x / \bar{y}_{l}\right)}, \quad x \geq 0 .
$$

2.2. Switch Model. Throughout the paper, a discrete-time model for the switch statistic and the SNR is used. In the MSSC system, at discrete-time $n$, let $\hat{l}(n)=l(l=1,2, \ldots, L)$ represent the event that branch $l$ is used and $\lambda_{l}(n)$ denote the general switch statistic. In the paper, the case where the switch statistic $\lambda_{l}(n)$ is a stationary process is considered.

For the MSSC system, the branch switching is characterized by the rule:

For $\hat{l}(n-1)=l, \quad \hat{l}(n)= \begin{cases}l, & \text { if } \lambda_{l}(n) \geq \eta_{l}, \\ l+1, & \text { if } \lambda_{l}(n)<\eta_{l}, l \leq L-1, \\ 1, & \text { if } \lambda_{l}(n)<\eta_{l}, l=L,\end{cases}$

where $\eta_{l} \geq 0$ is the designated ST. In the traditional SSC system [9-12], the faded SNR is employed as the switch statistic, that is, $\lambda_{l}(n)=\gamma_{l}(n)$. In the following analysis, the time index $n$ will be often omitted to simplify the notation.

The multibranch switch behavior can be modeled by the $L$-state stationary Markov chain as illustrated in Figure 1, where state $l(l=1,2, \ldots, L)$ means that branch $l$ is used for signal reception, and $p^{i, j}(i, j=1,2, \ldots, L)$ denotes the state transition probability. 




Figure 1: The $L$-state Markov chain for the switch model of the MSSC.

Under the condition that $s_{m}(m=0,1)$ has been transmitted, the transition probability of this Markov chain from state $i$ to state $j$ is given by

$$
p_{m}^{i, j}\left(\eta_{i}\right)= \begin{cases}P_{m}\left(\lambda_{i} \geq \eta_{i}\right), & j=i ; i=1,2, \ldots, L \\ P_{m}\left(\lambda_{i}<\eta_{i}\right), & j=i+1 ; i=1,2, \ldots, L-1, \\ P_{m}\left(\lambda_{i}<\eta_{i}\right), & i=L, j=1, \\ 0, & \text { otherwise. }\end{cases}
$$

Let $\pi_{l}(i=1,2, \ldots, L)$ be the stationary distribution of state $l$ of the Markov chain. With the above state transition probability, it can be shown that $\pi_{l}$ is a function of $\left(\eta_{1}, \eta_{2}, \ldots, \eta_{L}\right)$ and is given by

$$
\pi_{m, l}\left(\eta_{1}, \eta_{2}, \ldots, \eta_{L}\right)=\left(\sum_{j=1}^{L} \frac{1}{P_{m}\left(\lambda_{j}<\eta_{j}\right)}\right)^{-1} \frac{1}{P_{m}\left(\lambda_{l}<\eta_{l}\right)}
$$

Notice that if $\lambda_{1}, \lambda_{2}, \ldots, \lambda_{L}$ are i.i.d. with $\eta_{l}=\eta(l=1,2, \ldots$, $L)$, then $\pi_{m, l}=1 / L$ for all $l$.

\section{Performance Analysis}

To simplify the referring in the context, the three MSSC schemes are represented by the $|r|$-MSSC, $\mid$ ar $\mid$-MSSC, and $(a+|r|)$-MSSC, respectively. Throughout the paper, equally likely signaling is considered.

3.1. General BER Form. Let $P_{0}(\cdot)$ be the error probability under the condition that $s_{0}$ has been transmitted. Then, with the stationary distribution under fixed $\left(\eta_{1}, \eta_{2}, \ldots, \eta_{L}\right)$, the average BER of BPSK is given by

$$
P_{e}\left(\eta_{1}, \eta_{2}, \ldots, \eta_{L}\right)=\sum_{l=1}^{L} \pi_{0, l}\left(\eta_{1}, \eta_{2}, \ldots, \eta_{L}\right) P_{e, 0}\left(\eta_{l} \mid l\right)
$$

where $P_{e, 0}\left(\eta_{l} \mid l\right)=P_{0}$ (error $\left.\mid \hat{l}(n-1)=l\right)$ denotes the BER conditioned on the event that branch $l$ has been used in the previous discrete-time slot. For BPSK with equally likely signaling, the conditional BER $P_{e, 0}\left(\eta_{l} \mid l\right)$ has the form:

$$
\begin{aligned}
& P_{e, 0}\left(\eta_{l} \mid l\right) \\
& =P_{0}\left(\{\text { error with branch } l\} \operatorname{AND}\left\{\lambda_{l} \geq \eta_{l}\right\}\right) \\
& \quad+P_{0}\left(\{\text { error with branch } l+1, l \leq L-1\} \operatorname{AND}\left\{\lambda_{l}<\eta_{l}\right\}\right) \\
& \quad+P_{0}\left(\{\text { error with branch } 1, l=L\} \operatorname{AND}\left\{\lambda_{l}<\eta_{l}\right\}\right) \\
& =P_{0}\left(r_{l}>0, \lambda_{l} \geq \eta_{l}\right)+P_{0}\left(r_{[l]_{L}+1}>0\right) P_{0}\left(\lambda_{l}<\eta_{l}\right),
\end{aligned}
$$

where the notations of sets and "AND" operations are simplified, $[l]_{L}$ means $l$-modulo- $L$ for $0 \leq[l]_{L} \leq L-$ 1, $P_{0}$ (error with branch $l, \lambda_{1} \geq \eta_{1}$ ) is the error probability of using the old branch, and $P_{0}$ (error with branch $[l]_{L}+$ 1) is the error probability of using the new switched-to branch.

In the following BER analysis, we will first formulate the stationary distribution $\pi_{0, l}$ and the BER for fixed SNRs and then derive the average BER for faded SNRs.

3.2. $\mid$ ar $\mid-M S S C$. In the $\mid$ ar $\mid$-MSSC system, the switch statistic $\lambda_{l}=\left|a_{l} r_{l}\right|$ is used. This statistic has been applied to the optimal SC [14] that yields a minimal BER among different SC schemes. Thus, it will be expected that the receiver with the $\mid$ ar $\mid$-MSSC will yield the smallest BER compared to other MSSC schemes, which will be confirmed in the Section 5.

Under the condition that $s_{0}$ has been transmitted, the stationary distribution given by (6) for the $\mid$ ar $\mid$-MSSC has the form:

$$
\begin{aligned}
\pi_{0, l}\left(\eta_{1}, \eta_{2}, \ldots, \eta_{L}\right)= & \left(\sum_{j=1}^{L} \frac{1}{P_{0}\left(\left|a_{j} r_{j}\right|<\eta_{j}\right)}\right)^{-1} \\
& \times \frac{1}{P_{0}\left(\left|a_{l} r_{l}\right|<\eta_{l}\right)}
\end{aligned}
$$

where $\lambda_{l}=\left|a_{l} r_{l}\right|$. By defining the normalized threshold $\bar{\eta}_{j}=$ $\eta_{j} \sqrt{E_{b}} / N_{0}$, the probability $P_{0}\left(\left|a_{l} r_{l}\right|<\eta_{l}\right)$ in (9) can be written in the form:

$$
\begin{aligned}
P_{0}\left(\left|a_{j} r_{j}\right|<\eta_{j}\right)= & P_{0}\left(-\eta_{j}<a_{j} r_{j}<\eta_{j}\right) \\
= & -\int_{0}^{\infty} \frac{1}{2} \operatorname{erfc}\left(\sqrt{x}+\frac{\bar{\eta}_{j}}{\sqrt{x}}\right) f_{\gamma_{j}}(x) d x \\
& +\int_{0}^{\infty} \frac{1}{2} \operatorname{erfc}\left(\sqrt{x}-\frac{\bar{\eta}_{j}}{\sqrt{x}}\right) f_{\gamma_{j}}(x) d x
\end{aligned}
$$


where $\operatorname{erfc}(\cdot)$ is the complementary error function. For evaluating the integrals in (10), using integration by parts, we can evaluate the integral $\int_{0}^{\infty} \operatorname{erfc}\left(\sqrt{x}+\bar{\eta}_{j} / \sqrt{x}\right) f_{\gamma_{j}}(x) d x$ as

$$
\begin{aligned}
\int_{0}^{\infty} \operatorname{erfc}\left(\sqrt{x}+\frac{\bar{\eta}_{j}}{\sqrt{x}}\right) f_{\gamma_{j}}(x) d x \\
=\int_{0}^{\infty} \operatorname{erfc}\left(\sqrt{x}+\frac{\bar{\eta}_{j}}{\sqrt{x}}\right) \frac{1}{\bar{\gamma}_{l}} \exp \left(-\frac{x}{\bar{\gamma}_{l}}\right) d x \\
=\int_{0}^{\infty}\left[\frac{d}{d x} \operatorname{erfc}\left(\sqrt{x}+\frac{\bar{\eta}_{j}}{\sqrt{x}}\right)\right] \exp \left(-\frac{x}{\bar{\gamma}_{l}}\right) d x \\
=-\frac{e^{-2 \bar{\eta}_{j}}}{\sqrt{\pi}} \int_{0}^{\infty} x^{-1 / 2} e^{-\left(\left(\left(\bar{\gamma}_{l}+1\right) / \bar{\gamma}_{l}\right) x+\bar{\eta}_{j} / x\right)} d x \\
\quad+\frac{a \cdot e^{-2 \bar{\eta}_{j}}}{\sqrt{\pi}} \int_{0}^{\infty} x^{-3 / 2} e^{-\left(\left(\left(\bar{\gamma}_{l}+1\right) / \bar{\gamma}_{l}\right) x+\bar{\eta}_{j} / x\right)} d x,
\end{aligned}
$$

where, by applying [19, equation (3.472.3)], the two integrals $\int_{0}^{\infty} x^{-1 / 2} e^{-\left[\left(\bar{\gamma}_{l}+1\right) x / \bar{y}_{l}+\bar{\eta}_{j} / x\right]} d x$ and $\int_{0}^{\infty} x^{-3 / 2} e^{-\left[\left(\bar{y}_{l}+1\right) x / \bar{y}_{l}+\bar{y}_{j} / x\right]} d x$ can be derived and written in following closed-forms:

$$
\begin{gathered}
\int_{0}^{\infty} x^{-1 / 2} e^{-\left(\left(\left(\bar{\gamma}_{l}+1\right) / \bar{\gamma}_{l}\right) x+\bar{\eta}_{j} / x\right)} d x=\sqrt{\frac{\pi \bar{\gamma}_{l}}{\bar{\gamma}_{l}+1}} \cdot e^{-2 \bar{\eta}_{j} \sqrt{\left(\bar{\gamma}_{l}+1\right) / \bar{\gamma}_{l}}}, \\
\int_{0}^{\infty} x^{-3 / 2} e^{-\left(\left(\left(\bar{\gamma}_{l}+1\right) / \bar{\gamma}_{l}\right) x+\bar{\eta}_{j} / x\right)} d x=\frac{\sqrt{\pi}}{a} \cdot e^{-2 \bar{\eta}_{j} \sqrt{\left(\bar{\gamma}_{l}+1\right) / \bar{\gamma}_{l}}}
\end{gathered}
$$

Therefore,

$$
\begin{aligned}
& \int_{0}^{\infty} \frac{1}{2} \operatorname{erfc}\left(\sqrt{x}+\frac{\bar{\eta}_{j}}{\sqrt{x}}\right) f_{\gamma_{j}}(x) d x \\
& =\frac{1}{2}\left(1-\sqrt{\frac{\bar{\gamma}_{j}}{\bar{\gamma}_{j}+1}}\right) e^{-2 \bar{\eta}_{j}\left(\sqrt{1+1 / \bar{\gamma}_{j}}+1\right)} .
\end{aligned}
$$

Similarly, the first integral in (10) can also be derived and written in closed-form as

$$
\begin{aligned}
\int_{0}^{\infty} \frac{1}{2} \operatorname{erfc}\left(\sqrt{x}-\frac{\bar{\eta}_{j}}{\sqrt{x}}\right) \frac{1}{\bar{\gamma}_{l}} \exp \left(-\frac{x}{\bar{\gamma}_{l}}\right) d x \\
=1-\frac{e^{2 \bar{\eta}_{j}}}{2 \sqrt{\pi}} \int_{0}^{\infty} x^{-1 / 2} e^{-\left(\left(\left(\bar{\gamma}_{l}+1\right) / \bar{\gamma}_{l}\right) x+\bar{\eta}_{j} / x\right)} d x \\
\quad-\frac{\bar{\eta}_{j} \cdot e^{2 \bar{\eta}_{j}}}{2 \sqrt{\pi}} \int_{0}^{\infty} x^{-3 / 2} e^{-\left(\left(\left(\bar{\gamma}_{l}+1\right) / \bar{\gamma}_{l}\right) x+\bar{\eta}_{j} / x\right)} d x \\
=1-\frac{1}{2}\left(1-\sqrt{\frac{\bar{\gamma}_{l}}{\bar{\gamma}_{l}+1}}\right) e^{-2 \bar{\eta}_{j}\left(\sqrt{1+1 / \bar{\gamma}_{l}}-1\right)},
\end{aligned}
$$

where [19, equation (3.472.3)] is used again. Consequently, in (9), we have

$$
\begin{aligned}
P_{0}\left(\left|a_{j} r_{j}\right|<\eta_{j}\right)= & -\frac{1}{2}\left(1-\sqrt{\frac{\bar{\gamma}_{l}}{\bar{\gamma}_{l}+1}}\right) e^{-2 \bar{\eta}_{j}\left(\sqrt{1+1 / \bar{\gamma}_{l}}-1\right)} \\
& -\frac{1}{2}\left(1-\sqrt{\frac{\bar{\gamma}_{j}}{\bar{\gamma}_{j}+1}}\right) e^{-2 \bar{\eta}_{j}\left(\sqrt{\left.1+1 / \overline{\gamma_{j}}+1\right)}\right.} .
\end{aligned}
$$

For the $|a r|$-MSSC, the conditional BER $P_{e, 0}\left(\eta_{l} \mid l\right)$ in (8) is given by

$$
\begin{aligned}
P_{e, 0}\left(\eta_{l} \mid l\right)= & P_{0}\left(r_{l}>0, a_{l} r_{l} \geq \eta_{l}\right) \\
& +P_{0}\left(r_{[l]_{L}+1}>0\right) P_{0}\left(\left|a_{l} r_{l}\right|<\eta_{l}\right),
\end{aligned}
$$

where

$$
\begin{aligned}
P_{0}\left(r_{\left[l l_{L}+1\right.}>0\right) & =\int_{0}^{\infty} \frac{1}{2} \operatorname{erfc}\left(\sqrt{\gamma_{[l]_{L}+1}}\right) f_{\gamma_{\left[l l_{L}+1\right.}}(x) d x \\
& =\frac{1}{2}-\frac{1}{2} \sqrt{\frac{\bar{\gamma}_{\left[l_{L}+1\right.}}{\bar{\gamma}_{[]_{L}+1}+1}},
\end{aligned}
$$

and, from similar manipulations given by (11)-(13),

$$
\begin{aligned}
P_{0}\left(r_{l}>0, a_{l} r_{l} \geq \eta_{l}\right) & =P_{0}\left(a_{l} r_{l} \geq \eta_{l}\right) \\
& =\int_{0}^{\infty} \frac{1}{2} \operatorname{erfc}\left(\sqrt{x}+\frac{\bar{\eta}_{l}}{\sqrt{x}}\right) f_{\gamma_{l}}(x) d x \\
& =\frac{1}{2}\left(1-\sqrt{\frac{\bar{\gamma}_{l}}{\bar{\gamma}_{l}+1}}\right) e^{-2 \bar{\eta}_{l}\left(\sqrt{1+1 / \bar{\gamma}_{l}}+1\right)} .
\end{aligned}
$$

Based on (7) and (10)-(19), the BER of the $\mid$ ar $\mid$-MSSC can be obtained and written in the closed-form:

$$
\begin{aligned}
& P_{e}\left(\bar{\eta}_{1}, \bar{\eta}_{2}, \ldots, \bar{\eta}_{L}\right) \\
& =\frac{1}{2} \sum_{l=1}^{L}\left(1-\sqrt{\frac{\bar{\gamma}_{l}}{\bar{\gamma}_{l}+1}}\right)\left(\sum _ { j = 1 } ^ { L } \left[1-\frac{1}{2}\left(1+\sqrt{\frac{\bar{\gamma}_{j}}{\bar{\gamma}_{j}+1}}\right) e^{-2 \bar{\eta}_{j}\left(\sqrt{1+1 / \bar{\gamma}_{j}}-1\right)}\right.\right. \\
& \left.\left.-\frac{1}{2}\left(1-\sqrt{\frac{\bar{\gamma}_{j}}{\bar{\gamma}_{j}+1}}\right) e^{-2 \bar{\eta}_{j}\left(\sqrt{1+1 / \bar{\gamma}_{j}}+1\right)}\right]^{-1}\right)^{-1} \\
& \times\left\{1+e^{-2 \bar{\eta}_{l}\left(1+\sqrt{1+1 / \bar{\gamma}_{l}}\right)}\right. \\
& {\left[1-\frac{1}{2}\left(1+\sqrt{\frac{\bar{\gamma}_{l}}{\bar{\gamma}_{l}+1}}\right) e^{-2 \bar{\eta}_{l}\left(\sqrt{1+1 / \bar{\gamma}_{l}}-1\right)}\right.}
\end{aligned}
$$

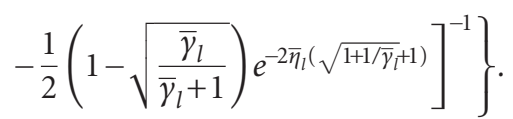

For i.i.d. fading channels with $\bar{\gamma}_{l}=\bar{\gamma}$ and identical ST $\bar{\eta}_{l}=\bar{\eta}$ for $l=1,2, \ldots, L$, the BER reduces to the form:

$$
\begin{aligned}
& P_{e}(\bar{\eta})=\frac{1}{2}\left(1-\sqrt{\frac{\bar{\gamma}}{\bar{\gamma}+1}}\right) \\
& \times\left[1+e^{-2 \bar{\eta}(\sqrt{1+1 / \bar{\gamma}}+1)}\right. \\
&-\frac{1}{2}\left(1+\sqrt{\frac{\bar{\gamma}}{\bar{\gamma}+1}}\right) e^{-2 \bar{\eta}(\sqrt{1+1 / \bar{\gamma}}-1)} \\
&\left.-\frac{1}{2}\left(1-\sqrt{\frac{\bar{\gamma}}{\bar{\gamma}+1}}\right) e^{-2 \bar{\eta}(\sqrt{1+1 / \bar{\gamma}}+1)}\right] .
\end{aligned}
$$


For the i.i.d. channels with $\bar{\eta}=0$ since we always have $\left|a_{l} r_{l}\right| \geq 0(l=1,2, \ldots, L)$ and the switch will not occur (i.e., no SSC is used), the above BER reduces to the form:

$$
P_{e}=\frac{1}{2}-\frac{1}{2} \sqrt{\frac{\bar{\gamma}}{\bar{\gamma}+1}} \text {. }
$$

The BER given by (22) agrees with the well-known average BER of BPSK on a Rayleigh fading channel without diversity combining [20].

From (21) for the i.i.d. channels, the BER performance remains the same for any value of $L \geq 2$, and the BER cannot be improved by adding more diversity branches.

3.3. $(a+|r|)$-MSSC. To save the processing time and power in an MSSC system and maintain its performance, other combination of $a$ and $|r|$ may be considered. Notice that the $\mid$ ar $\mid$-MSSC given in the previous subsection uses a multiplier which is more time- and power-consuming than an adder. In the $(a+|r|)$-MSSC system, the switch statistic is $\lambda_{l}=$ $a_{l} \sqrt{E_{b}}+\left|r_{l}\right|$, where an adder rather than a multiplier is used and $\sqrt{E_{b}}$ is a scaling constant. In the section of numerical results, it will be shown that $(a+|r|)$-MSSC and $|a r|$-MSSC have similar performance, which is significantly better than the SNR-based SSC.

With the switch statistic $\lambda_{l}=a_{l} \sqrt{E_{b}}+\left|r_{l}\right|$ for $l=$ $1,2, \ldots, L$, the relevant probability in (6) now is given by $P_{0}\left(a_{j} \sqrt{E_{b}}+\left|r_{j}\right|<\eta_{j}\right)$. For $(a+|r|)$-MSSC, defining another normalized ST as $\tilde{\eta}_{j}=\eta_{j} / \sqrt{N_{0}}$, we have

$$
\begin{aligned}
& P_{0}\left(a_{j} \sqrt{E_{b}}+\left|r_{j}\right|<\eta_{j}\right) \\
& =P_{0}\left(-\eta_{j}+a_{j} \sqrt{E_{b}}<r_{j}<\eta_{j}-a_{j} \sqrt{E_{b}}\right) \\
& =P_{0}\left(n_{j}<\eta_{j}\right)-P_{0}\left(n_{j}<2 a_{j} \sqrt{E_{b}}-\eta_{j}\right) \\
& =\int_{0}^{\tilde{\eta}_{j}^{2}} \frac{1}{2} \operatorname{erfc}\left(2 \sqrt{x}-\tilde{\eta}_{j}\right) d x-\frac{1}{2} \operatorname{erfc}\left(\tilde{\eta}_{j}\right),
\end{aligned}
$$

where the upper limit $\tilde{\eta}_{j}^{2}$ of the integral comes from the intrinsic constraint associated with the set $\left\{-\eta_{j}+a_{j} \sqrt{E_{b}}<\right.$ $\left.r_{j}<\eta_{j}-a_{j} \sqrt{E_{b}}\right\}$. To evaluate the integral in (23), we use integration by parts to obtain

$$
\begin{aligned}
\int_{0}^{\tilde{\eta}_{j}^{2}} \frac{1}{2} \operatorname{erfc}\left(2 \sqrt{x}-\tilde{\eta}_{j}\right) d x \\
=\frac{1}{2} \operatorname{erfc}\left(-\tilde{\eta}_{j}\right)-\frac{e^{-\tilde{\eta}_{j}^{2} / \bar{\gamma}_{j}}}{2} \operatorname{erfc}\left(\tilde{\eta}_{j}\right) \\
\quad-\int_{0}^{\tilde{\eta}_{j}^{2}} \frac{1}{\sqrt{\pi x}} e^{-\left(2 \sqrt{x}-\tilde{\eta}_{j}\right)^{2}-x / \bar{\gamma}_{l}} d x
\end{aligned}
$$

where it is straightforward to show that

$$
\begin{aligned}
\int_{0}^{\tilde{\eta}_{j}^{2}} \frac{1}{\sqrt{\pi x}} e^{-\left(2 \sqrt{x}-\tilde{\eta}_{j}\right)^{2}-x / \bar{\gamma}_{l}} d x & \sqrt{\frac{\bar{\gamma}_{l}}{4 \bar{\gamma}_{l}+1}} e^{-\left(-\tilde{\eta}_{j}^{2} /\left(4 \bar{\gamma}_{l}+1\right)\right)} \\
\times & {\left[\frac { 1 } { 2 } \operatorname { e r f c } \left(-2 \tilde{\eta}_{j} \sqrt{\left.\frac{\bar{\gamma}_{l}}{4 \bar{\gamma}_{l}+1}\right)}\right.\right.} \\
& \left.\quad-\frac{1}{2} \operatorname{erfc}\left(\sqrt{\frac{\tilde{\eta}_{j}^{2}\left(4 \bar{\gamma}_{l}+1\right)}{\bar{\gamma}_{l}}}-2 \tilde{\eta}_{j} \sqrt{\frac{\bar{\gamma}_{l}}{4 \bar{\gamma}_{l}+1}}\right)\right] .
\end{aligned}
$$

Consequently,

$$
\begin{aligned}
P_{0}\left(a_{j} \sqrt{E_{b}}+\left|r_{j}\right|<\eta_{j}\right) \\
=1-\operatorname{erfc}\left(\tilde{\eta}_{j}\right)-\frac{e^{-\tilde{\eta}_{j}^{2} / \bar{\gamma}_{j}}}{2} \operatorname{erfc}\left(\tilde{\eta}_{j}\right) \\
-2 \sqrt{\frac{\bar{\gamma}_{l}}{4 \bar{\gamma}_{l}+1}} e^{-\left(-\tilde{\eta}_{j}^{2} /\left(4 \bar{\gamma}_{l}+1\right)\right)} \\
\times\left[\frac { 1 } { 2 } \operatorname { e r f c } \left(-2 \tilde{\eta}_{j} \sqrt{\left.\frac{\bar{\gamma}_{l}}{4 \bar{\gamma}_{l}+1}\right)}\right.\right. \\
\left.\quad-\frac{1}{2} \operatorname{erfc}\left(\sqrt{\frac{\tilde{\eta}_{j}^{2}\left(4 \bar{\gamma}_{l}+1\right)}{\bar{\gamma}_{l}}}-2 \tilde{\eta}_{j} \sqrt{\frac{\bar{\gamma}_{l}}{4 \bar{\gamma}_{l}+1}}\right)\right] .
\end{aligned}
$$

For the $(a+|r|)$-MSSC system, the conditional BER $P_{e}\left(\eta_{l} \mid l\right)$ in $(8)$ now is given by

$$
\begin{aligned}
P_{e}\left(\eta_{l} \mid l\right)= & \operatorname{Pr}\left(n_{l}>a_{l} \sqrt{E_{b}}, n_{l} \geq \eta_{l}\right) \\
& +P_{0}\left(r_{[l]_{L}+1}>0\right) P_{0}\left(\alpha_{l} \sqrt{E_{b}}+\left|r_{l}\right|<\eta_{l}\right),
\end{aligned}
$$

where

$$
\begin{aligned}
\operatorname{Pr}\left(n_{l}>a_{l} \sqrt{E_{b}}, n_{l} \geq \eta_{l}\right) \\
= \begin{cases}\operatorname{Pr}\left(n_{l}>\alpha_{l} \sqrt{E_{b}}\right), & \alpha_{l} \sqrt{E_{b}}>\eta_{l} \\
\operatorname{Pr}\left(n_{l}>\eta_{l}\right), & \alpha_{l} \sqrt{E_{b}} \leq \eta_{l}\end{cases} \\
=\int_{0}^{\widetilde{\eta}_{l}^{2}} \frac{1}{2} \operatorname{erfc}(x) f_{\gamma_{l}}(x) d x+\int_{\widetilde{\eta}_{l}^{2}}^{\infty} \frac{1}{2} \operatorname{erfc}(x) f_{\gamma_{l}}(x) d x \\
=\frac{1}{2} \operatorname{erfc}\left(\tilde{\eta}_{l}\right)-\frac{1}{2} \sqrt{\frac{\bar{\gamma}_{l}}{\bar{\gamma}_{l}+1}} \cdot \operatorname{erfc}\left(\tilde{\eta}_{l} \sqrt{\frac{\bar{\gamma}_{l}+1}{\bar{\gamma}_{l}}}\right),
\end{aligned}
$$


and $P_{0}\left(r_{[l]_{L}+1}>0\right)$ and $P_{0}\left(\alpha_{l} \sqrt{E_{b}}+\left|r_{l}\right|<\eta_{l}\right)$ have been already specified in (18) and (26), respectively.

Based on (7) and (23)-(27), the BER can be derived and written in the closed-form:

$$
\begin{aligned}
& P_{e}\left(\tilde{\eta}_{1}, \tilde{\eta}_{2}, \ldots, \tilde{\eta}_{L}\right) \\
& =\left(\sum _ { j = 1 } ^ { L } \left\{1-\frac{2+e^{-\tilde{\eta}_{j}^{2} / \bar{\gamma}_{j}}}{2} \operatorname{erfc}\left(\tilde{\eta}_{j}\right)-\sqrt{\frac{\bar{\gamma}_{j}}{4 \bar{\gamma}_{j}+1}} e^{-\widetilde{\eta}_{j}^{2} /\left(4 \bar{\gamma}_{j}+1\right)}\right.\right. \\
& \times\left[\operatorname{erfc}\left(-\tilde{\eta}_{j} \sqrt{\frac{4 \bar{\gamma}_{j}}{4 \bar{\gamma}_{j}+1}}\right)\right. \\
& \left.\left.\left.-\operatorname{erfc}\left(\frac{\left(2 \bar{\gamma}_{j}+1\right) \tilde{\eta}_{j}}{\sqrt{\bar{\gamma}_{j}\left(4 \bar{\gamma}_{j}+1\right)}}\right)\right]\right\}^{-1}\right)^{-1} \\
& \times \sum_{l=1}^{L}\left[\frac{1}{2} \operatorname{erfc}\left(\tilde{\eta}_{l}\right)-\frac{1}{2} \sqrt{\frac{\bar{\gamma}_{l}}{\bar{\gamma}_{l}+1}} \operatorname{erfc}\left(\tilde{\eta}_{l} \sqrt{\frac{\bar{\gamma}_{l}+1}{\bar{\gamma}_{l}}}\right)\right] \\
& \times\left\{1-\frac{2+e^{-\widetilde{\eta}_{l}^{2} / \bar{y}_{l}}}{2} \operatorname{erfc}\left(\tilde{\eta}_{l}\right)-\sqrt{\frac{\bar{\gamma}_{l}}{4 \bar{\gamma}_{l}+1}} e^{-\widetilde{\eta}_{l}^{2} /\left(4 \bar{\gamma}_{l}+1\right)}\right. \\
& \left.\times\left[\operatorname{erfc}\left(-\tilde{\eta}_{l} \sqrt{\frac{4 \bar{\gamma}_{l}}{4 \bar{\gamma}_{l}+1}}\right)-\operatorname{erfc}\left(\frac{\left(2 \bar{\gamma}_{l}+1\right) \tilde{\eta}_{l}}{\sqrt{\bar{\gamma}_{l}\left(4 \bar{\gamma}_{l}+1\right)}}\right)\right]\right\}^{-1} \\
& +\left(\sum _ { j = 1 } ^ { L } \left\{1-\frac{2+e^{-\tilde{\eta}_{j}^{2} / \bar{\gamma}_{j}}}{2} \operatorname{erfc}\left(\tilde{\eta}_{j}\right)-\sqrt{\frac{\bar{\gamma}_{j}}{4 \bar{\gamma}_{j}+1}} e^{-\tilde{\eta}_{j}^{2} /\left(4 \bar{\gamma}_{j}+1\right)}\right.\right. \\
& \times\left[\operatorname{erfc}\left(-\tilde{\eta}_{j} \sqrt{\frac{4 \bar{\gamma}_{j}}{4 \bar{\gamma}_{j}+1}}\right)\right. \\
& \left.\left.\left.-\operatorname{erfc}\left(\frac{\left(2 \bar{\gamma}_{j}+1\right) \tilde{\eta}_{j}}{\sqrt{\bar{\gamma}_{j}\left(4 \bar{\gamma}_{j}+1\right)}}\right)\right]\right\}^{-1}\right)^{-1} \\
& \times \sum_{l=1}^{L} \frac{1}{2}\left(1-\sqrt{\frac{\bar{\gamma}_{l}}{\bar{\gamma}_{l}+1}}\right) .
\end{aligned}
$$

With $\tilde{\eta}_{l}=\tilde{\eta}$, (29) reduces to the following form for i.i.d. fading channels:

$$
\begin{aligned}
P_{e}(\tilde{\eta})= & \frac{1}{2} \operatorname{erfc}(\tilde{\eta})-\frac{1}{2} \sqrt{\frac{\bar{\gamma}}{\bar{\gamma}+1}} \operatorname{erfc}\left(\sqrt{\frac{\bar{\gamma}+1}{\bar{\gamma}}} \tilde{\eta}\right) \\
& +\frac{1}{2}\left(1-\sqrt{\frac{\bar{\gamma}}{\bar{\gamma}+1}}\right)\left(1-\frac{2+e^{-\tilde{\eta}_{j}^{2} / \bar{\gamma}_{j}}}{2} \operatorname{erfc}(\widetilde{\eta})\right)
\end{aligned}
$$

$$
\begin{aligned}
& -\frac{1}{2}\left(1-\sqrt{\frac{\bar{\gamma}}{\bar{\gamma}+1}}\right) \sqrt{\frac{\bar{\gamma}}{4 \bar{\gamma}+1}} e^{-\widetilde{\eta}_{j}^{2} /\left(4 \bar{\gamma}_{j}+1\right)} \\
& \times\left[\operatorname{erfc}\left(-2 \tilde{\eta} \sqrt{\frac{\bar{\gamma}}{4 \bar{\gamma}+1}}\right)-\operatorname{erfc}\left(\frac{(2 \bar{\gamma}+1) \tilde{\eta}}{\sqrt{\bar{\gamma}(4 \bar{\gamma}+1)}}\right)\right] .
\end{aligned}
$$

From (30) for i.i.d. channels, the performance remains the same even if more than two branches are used. Again, if no SSC is activated by setting $\tilde{\eta}=0$ in (30), it is easy to recognize that (30) becomes the form given by (22) for the situation without SSC.

3.4. $|r|$-MSSC. To further reduce implementation complexity, $\left|r_{l}\right|$ can also be considered as the switch statistic of the MSSC, that is, $\lambda_{l}=\left|r_{l}\right|$. The corresponding probability in the stationary distribution given by (6) for the $|r|$-MSSC has the form:

$$
\begin{aligned}
P_{0}\left(\left|r_{j}\right|\right. & \left.<\eta_{j}\right) \\
= & P_{0}\left(n_{j}<a_{j} \sqrt{E_{b}}+\eta_{j}\right)-P_{0}\left(n_{j}<a_{j} \sqrt{E_{b}}-\eta_{j}\right) \\
= & -\int_{0}^{\infty} \frac{1}{2} \operatorname{erfc}\left(\sqrt{x}+\frac{\eta_{j}}{\sqrt{N_{0}}}\right) f_{\gamma_{j}}(x) d x \\
& +\int_{0}^{\infty} \frac{1}{2} \operatorname{erfc}\left(\sqrt{x}-\frac{\eta_{j}}{\sqrt{N_{0}}}\right) f_{\gamma_{j}}(x) d x .
\end{aligned}
$$

To evaluate (31), following steps similar to (24) and (25), we have

$$
\begin{aligned}
\int_{0}^{\infty} \frac{1}{2} \operatorname{erfc}\left(\sqrt{x} \pm \tilde{\eta}_{j}\right) f_{\gamma_{j}}(x) d x \\
=\frac{1}{2} \operatorname{erfc}\left( \pm \tilde{\eta}_{j}\right)-\frac{1}{2} \sqrt{\frac{\bar{\gamma}_{l}}{\bar{\gamma}_{l}+1}} \\
\quad \times e^{-\left(-\tilde{\eta}_{j}^{2} /\left(\bar{\gamma}_{l}+1\right)\right)} \operatorname{erfc}\left( \pm \tilde{\eta}_{j} \sqrt{\frac{\bar{\gamma}_{l}}{\bar{\gamma}_{l}+1}}\right) .
\end{aligned}
$$

Consequently, the probability given by (31) turns into the form

$$
\begin{aligned}
P_{0}\left(\left|r_{j}\right|<\eta_{j}\right) & \\
= & 1-\operatorname{erfc}\left(\tilde{\eta}_{j}\right)-\sqrt{\frac{\bar{\gamma}_{j}}{\bar{\gamma}_{j}+1}} e^{-\left(-\tilde{\eta}_{j}^{2} /\left(\bar{\gamma}_{l}+1\right)\right)}+\sqrt{\frac{\bar{\gamma}_{j}}{4 \bar{\gamma}_{j}+1}} \\
& \times e^{-\left(-\tilde{\eta}_{j}^{2} /\left(\bar{\gamma}_{l}+1\right)\right)} \operatorname{erfc}\left(\tilde{\eta}_{j} \sqrt{\frac{\bar{\gamma}_{j}}{\bar{\gamma}_{j}+1}}\right) .
\end{aligned}
$$

The conditional BER $P_{e}\left(\eta_{l} \mid l\right)$ given by (8) now has the form

$$
P_{e}\left(\eta_{l} \mid l\right)=P_{0}\left(r_{l}>0, r_{l} \geq \eta_{l}\right)+P_{0}\left(r_{\left[l_{L}+1\right.}>0\right) P_{0}\left(\left|r_{l}\right|<\eta_{l}\right),
$$


where $P_{0}\left(r_{[]_{L}+1}>0\right)$ has been given in (18) and based on (32):

$$
\begin{aligned}
P_{0}\left(r_{l}>0, r_{l} \geq \eta_{l}\right)= & P_{0}\left(r_{l} \geq \eta_{l}\right) \\
= & \int_{0}^{\infty} \frac{1}{2} \operatorname{erfc}\left(\sqrt{x}+\tilde{\eta}_{l}\right) f_{\gamma_{l}}(x) d x \\
= & \frac{1}{2} \operatorname{erfc}\left(\tilde{\eta}_{j}\right)-\frac{1}{2} \sqrt{\frac{\bar{\gamma}_{l}}{\bar{\gamma}_{l}+1}} \\
& \times e^{-\left(-\tilde{\eta}_{j}^{2} /\left(\bar{\gamma}_{l}+1\right)\right)} \operatorname{erfc}\left(\tilde{\eta}_{j} \sqrt{\frac{\bar{\gamma}_{l}}{\bar{\gamma}_{l}+1}}\right) .
\end{aligned}
$$

In consequence, according to (7), (33), and (34), the BER is given by the closed-form:

$$
\begin{aligned}
& P_{e}\left(\tilde{\eta}_{1}, \tilde{\eta}_{2}, \ldots, \tilde{\eta}_{L}\right) \\
& =\sum_{l=1}^{L}\left(\frac{1}{2} \operatorname{erfc}\left(\tilde{\eta}_{l}\right)-\frac{e^{-\tilde{\eta}_{l}^{2} /\left(\bar{\gamma}_{l}+1\right)}}{2} \sqrt{\frac{\bar{\gamma}_{l}}{\bar{\gamma}_{l}+1}} \operatorname{erfc}\left(\tilde{\eta}_{l} \sqrt{\frac{\bar{\gamma}_{l}}{\bar{\gamma}_{l}+1}}\right)\right) \\
& \times\left(\sum _ { j = 1 } ^ { L } \left[1-e^{-\tilde{\eta}_{j}^{2} /\left(\bar{\gamma}_{j}+1\right)} \sqrt{\frac{\bar{\gamma}_{j}}{\bar{\gamma}_{j}+1}}+e^{-\tilde{\eta}_{j}^{2} /\left(\bar{\gamma}_{j}+1\right)} \sqrt{\frac{\bar{\gamma}_{j}}{\bar{\gamma}_{j}+1}}\right.\right. \\
& \left.\left.\cdot \operatorname{erfc}\left(\tilde{\eta}_{j} \sqrt{\frac{\bar{\gamma}_{j}}{\bar{\gamma}_{j}+1}}\right)-\operatorname{erfc}\left(\tilde{\eta}_{j}\right)\right]^{-1}\right)^{-1} \\
& \times\left[1-e^{-\widetilde{\eta}_{l}^{2} /\left(\bar{\gamma}_{l}+1\right)} \sqrt{\frac{\bar{\gamma}_{l}}{\bar{\gamma}_{l}+1}}+\frac{e^{-\tilde{\eta}_{l}^{2} /\left(\bar{\gamma}_{l}+1\right)}}{2} \sqrt{\frac{\bar{\gamma}_{l}}{\bar{\gamma}_{l}+1}}\right. \\
& \left.\cdot \operatorname{erfc}\left(\tilde{\eta}_{l} \sqrt{\frac{\bar{\gamma}_{l}}{\bar{\gamma}_{l}+1}}\right)-\operatorname{erfc}\left(\tilde{\eta}_{l}\right)\right]^{-1} \\
& +\sum_{l=1}^{L}\left(\frac{1}{2}-\frac{1}{2} \sqrt{\frac{\bar{\gamma}_{l}}{\bar{\gamma}_{l}+1}}\right) \\
& \times\left(\sum _ { j = 1 } ^ { L } \left[1-e^{-\tilde{\eta}_{j}^{2} /\left(\bar{\gamma}_{j}+1\right)} \sqrt{\frac{\bar{\gamma}_{j}}{\bar{\gamma}_{j}+1}}+e^{-\tilde{\eta}_{j}^{2} /\left(\bar{\gamma}_{j}+1\right)} \sqrt{\frac{\bar{\gamma}_{j}}{\bar{\gamma}_{j}+1}}\right.\right. \\
& \left.\left.\cdot \operatorname{erfc}\left(\tilde{\eta}_{j} \sqrt{\frac{\bar{\gamma}_{j}}{\bar{\gamma}_{j}+1}}\right)-\operatorname{erfc}\left(\tilde{\eta}_{j}\right)\right]^{-1}\right)^{-1} \cdot
\end{aligned}
$$

For i.i.d. fading channels with $\tilde{\eta}_{l}=\tilde{\eta}$, the above BER turns into the form:

$$
\begin{aligned}
P_{e}(\tilde{\eta})= & \left(\frac{1}{2}-\frac{1}{2} \sqrt{\frac{\bar{\gamma}}{\bar{\gamma}+1}}\right)\left(1-\sqrt{\frac{\bar{\gamma}}{\bar{\gamma}+1}} e^{-\tilde{\eta}^{2} /(\bar{\gamma}+1)}\right) \\
& -\frac{1}{2} \sqrt{\frac{\bar{\gamma}}{\bar{\gamma}+1}} \\
& \times\left[\sqrt{\frac{\bar{\gamma}}{\bar{\gamma}+1}} e^{-\tilde{\eta}^{2} /(\bar{\gamma}+1)} \operatorname{erfc}\left(\frac{\tilde{\eta}}{\sqrt{1+1 / \bar{\gamma}}}\right)-\operatorname{erfc}(\tilde{\eta})\right],
\end{aligned}
$$

that is also independent of $L$. If $\tilde{\eta}=0$ is substituted into (37) for the i.i.d. fading channels, the BER given by (37) also reduces to the form given by (22).

\section{Performance Optimization}

To minimize the average BER, the optimal ST is pursued for the three MSSC schemes in this section. To obtain the globally optimal ST for each branch, the $L$ simultaneous equations $\partial P_{e}\left(\tilde{\eta}_{1}, \tilde{\eta}_{2}, \ldots, \tilde{\eta}_{L}\right) / \partial \tilde{\eta}_{l}=0\left(\right.$ or $\partial P_{e}\left(\bar{\eta}_{1}, \bar{\eta}_{2}, \ldots, \bar{\eta}_{L}\right) / \partial \bar{\eta}_{l}=$ 0 if the $|a r|$-MSSC is used) for $l=1,2, \ldots, L$ have to be solved, which is difficult for a general case. Thus, we turn to the locally optimal ST. The locally optimal ST is defined as the ST that minimizes the conditional BER $P_{e}\left(\tilde{\eta}_{l} \mid l\right)$ or $P_{e}\left(\bar{\eta}_{l} \mid l\right)$ for each branch.

For the MSSC implementationin a receiver, a branch is randomly chosen at the beginning of a MSSC procedure, where the STs can be designated on a timeslot-by-timeslot basis. Under this consideration, the conditioned BER $P_{e}\left(\eta_{l} \mid\right.$ $l$ ) of branch $l$ for i.n.d. fading channels can be used as the object function to minimize for the determination of the locally optimal $\eta_{l}(l=1,2, \ldots, L)$.

For i.i.d. fading channels with an identical ST $\eta_{l}=\eta$ for $l=1,2, \ldots, L$, an interesting result is that the conditional BER is independent of $l$ and equals the total average BER, that is, $P_{e}\left(\bar{\eta}_{l} \mid l\right)=P_{e}(\bar{\eta})$ and $P_{e}\left(\widetilde{\eta}_{l} \mid l\right)=P_{e}(\tilde{\eta})$. In consequence, the locally optimal ST is also globally optimal for i.i.d. fading channels.

4.1. $|\operatorname{ar}|-$ MSSC. With the $|\operatorname{ar}|$-MSSC, the locally optimal ST for $l=1,2, \ldots, L$ can be obtained by solving $\partial P_{e}\left(\bar{\eta}_{l} \mid l\right) / \partial \bar{\eta}_{l}=$ 0 that gives the equation (after some manipulations):

$$
\left(1-\sqrt{\frac{\bar{\gamma}_{[]_{L}+1}}{\bar{\gamma}_{[]_{L}+1}+1}}\right) e^{2 \bar{\eta}_{l, o}}=\left(1+\sqrt{\frac{\bar{\gamma}_{[l]_{L}+1}}{\bar{\gamma}_{[l]_{L}+1}+1}}\right) e^{-2 \bar{\eta}_{l, o}},
$$

where $\bar{\eta}_{l}=\bar{\eta}_{l, 0}$ is the locally optimal ST. Solving (38) for $\bar{\eta}_{l, o}$, we obtain the locally optimal ST expressed in the closedform:

$$
\bar{\eta}_{l, o}=\frac{1}{4} \ln \left(\frac{\sqrt{\bar{\gamma}_{[]_{L}+1}+1}+\sqrt{\bar{\gamma}_{\left[l_{L}+1\right.}}}{\sqrt{\bar{\gamma}_{[]_{L}+1}+1}-\sqrt{\bar{\gamma}_{\left[l_{L}+1\right.}}}\right) .
$$

From (39), a surprising result is that the locally optimal ST of $\mid$ ar|-MSSC only depends on the average SNR of the switched-to branch.

As aforementioned, for i.i.d. fading channels with $\bar{\gamma}_{l}=\bar{\gamma}$ and $\bar{\eta}_{l}=\bar{\eta}$ for $l=1,2, \ldots, L, \bar{\eta}_{l, o}=\bar{\eta}_{o}$ also gives the globally optimal ST.

4.2. $(a+|r|)$-MSSC. In the $(a+|r|)$-MSSC system, the equation $\partial P_{e}\left(\tilde{\eta}_{l} \mid l\right) / \partial \tilde{\eta}_{l}=0$ is to be solved for the locally optimal ST for branch $l(l=1,2, \ldots, \mathrm{L})$, which leads to a complex nonlinear equation which can only be solved by a numerical method such as the one given in [21]. For the i.i.d. fading channels with an identical ST, the globally optimal ST $\tilde{\eta}_{o}$ can be derived from the nonlinear equation. 
4.3. $|r|$-MSSC. For the $|r|$-MSSC with the conditional BER given by (34) and the normalized threshold $\tilde{\eta}_{l}$, the equation $\partial P_{e}\left(\tilde{\eta}_{l} \mid l\right) / \partial \tilde{\eta}_{l}=0$ at the locally optimal ST $\tilde{\eta}_{l}=\tilde{\eta}_{l, o}$ yields

$$
\begin{gathered}
\operatorname{erfc}\left(\tilde{\eta}_{l, o} \sqrt{\left.\frac{\bar{\gamma}_{l}}{\bar{\gamma}_{l}+1}\right)+\frac{\sqrt{\bar{\gamma}_{[l]_{L}+1}+1}}{\sqrt{\bar{\gamma}_{[l]_{L}+1}}}-1}\right. \\
=\frac{1}{\sqrt{\pi}} \sqrt{\frac{\bar{\gamma}_{l}+1}{\bar{\gamma}_{l}}} \cdot \frac{e^{\left.-\bar{\gamma}_{l} \tilde{\eta}_{l, o}^{2} / \bar{\gamma}_{l}+1\right)}}{\tilde{\eta}_{l, o}},
\end{gathered}
$$

for $l=1,2, \ldots, L$. The nonlinear equation given by (40) can be easily solved by using a numerical method [21]. Again, for the i.i.d. fading channels, the globally optimal ST of the $|r|$ MSSC is obtained by solving (40).

\section{Numerical Results}

In this section, numerical results of BER based on the analysis are presented, and the results from Monte Carlo simulation are also supplied.

In Figure 2, the BERs of the three MSSC schemes on i.i.d. fading channels for $L=2$ are illustrated. As expected in Section 3, among the MSSC schemes, the $\mid$ ar $\mid$-MSSC yields the best performance. The $(a+|r|)$-MSSC and $|a r|$-MSSC schemes have similar performance, and both of them have a much smaller BER than the $|r|$-MSSC scheme. Furthermore, the $|a r|$-MSSC and $(a+|r|)$-MSSC schemes even have a smaller BER than SNR-based SC scheme for some ranges of $\bar{\gamma}$ (e.g., $\bar{\gamma} \leq 6 \mathrm{~dB}$ in Figure 2), where, unlike the MSSC, the $\mathrm{SC}$ requires power-consuming multibranch monitoring.

The BER of the SNR-based SSC is also plotted in Figure 2, where all the three MSSC schemes outperform the traditional SNR-based SSC and the power gain for the $|a r|$-MSSC or $(a+$ $|r|$ )-MSSC over the SNR-based SSC could be more than $2 \mathrm{~dB}$ for larger values of $\bar{\gamma}$.

The BER comparison on i.n.d. fading channels is illustrated in Figure 3, where corresponding locally optimal STs are used for the MSSC schemes, and two scenarios of the average SNRs are considered. Basically, three different average SNRs $\left(\bar{\gamma}_{1}, \bar{\gamma}_{2}, \bar{\gamma}_{3}\right)$ are used, where the relations $\bar{\gamma}_{2}=$ $\bar{\gamma}_{1} / 4$ and $\bar{\gamma}_{3}=\bar{\gamma}_{1} / 8$ are set. Thus, $\bar{\gamma}_{1}>\bar{\gamma}_{2}>\bar{\gamma}_{3}$. For the dualbranch case, two branches with $\bar{\gamma}_{2}$ and $\bar{\gamma}_{3}$ are used. For the triple-branch case, another branch with $\bar{\gamma}_{1}$ is added. Notice that the new added branch has the largest average SNR. From numerical results, we find that the performance of MSSC schemes can be enhanced if newly added branches have a larger average SNR than the used branches, which can be observed from Figure 3. On the other hand, if newly added branches do not have a larger average SNR, the resultant performance with more branches may be worse.

The BER of the SNR-based SC is also plotted in Figure 3 for performance comparisons. Based on these comparisons, the performance of both the $|a r|$-MSSC and $(a+|r|)$-MSSC schemes approaches and may be even better than that of the SNR-based SC scheme for some ranges of smaller average SNRs. For example, in the dual-branch case in Figure 3, both the $|a r|$-MSSC and $(a+|r|)$-MSSC schemes outperform the SNR-based SC scheme for $\bar{\gamma}_{1} \leq 14 \mathrm{~dB}$ (which is similar to the phenomenon on i.i.d. fading channels).

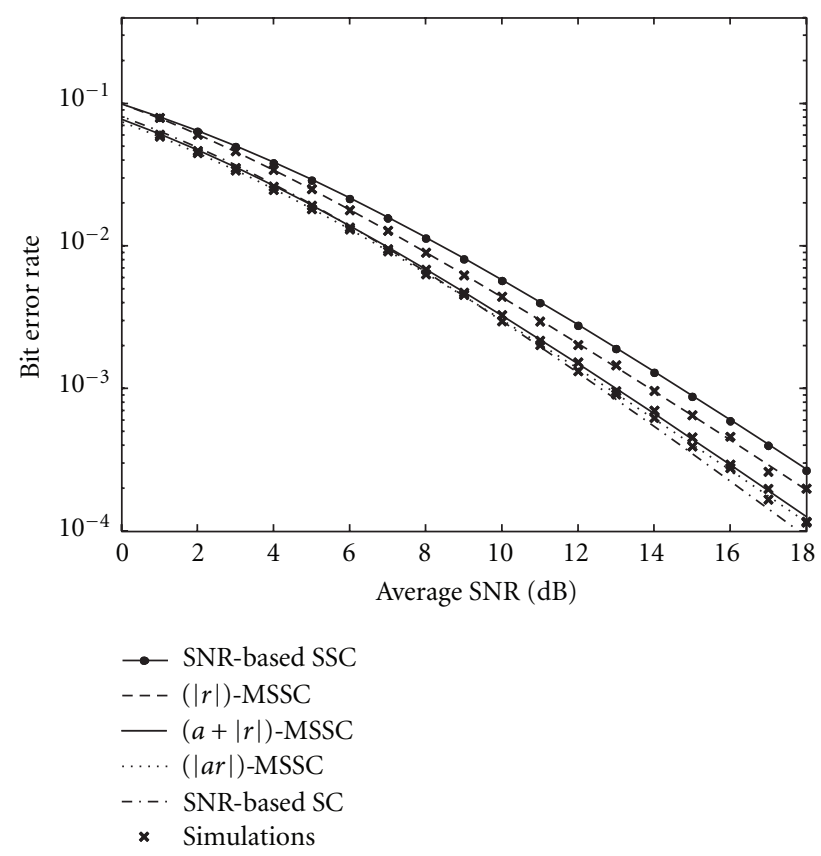

FIgURe 2: Performance comparisons for different dual-branch MSSC schemes on i.i.d. channels.

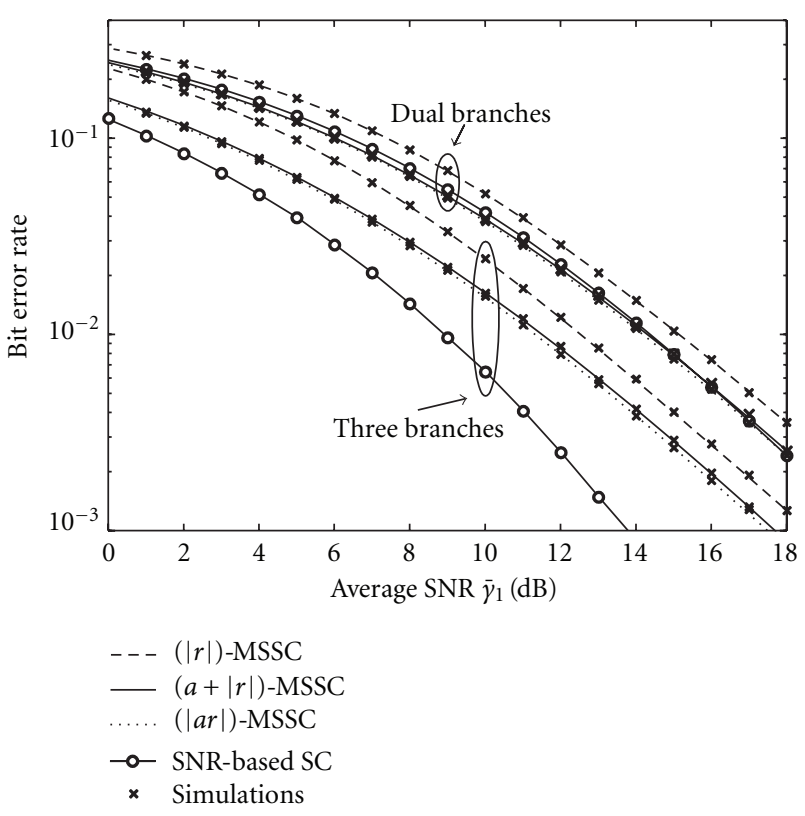

FIGURE 3: Performance comparisons for different MSSC schemes on i.n.d. channels.

\section{Conclusions}

Three MSSC schemes with new switch statistics have been developed and evaluated for both i.i.d. and i.n.d. fading channels, where the $\mid$ ar $\mid$-MSSC yields the best receiver performance but also has the highest implementation complexity. The $(a+|r|)$-MSSC and $|a r|$-MSSC schemes have similar performance. To generate the switch statistic, the 
$(a+|r|)$-MSSC uses an adder instead of the multiplier required by the $|a r|$-MSSC, which makes the $(a+|r|)$ MSSC faster and more power-saving than the $\mid$ ar $\mid$-MSSC. All the three new MSSC schemes outperform the traditional SNR-based SSC. The $|a r|$-MSSC and $(a+|r|)$-MSSC may even have a lower BER than the traditional SNR-based SC that requires simultaneous multibranch monitoring. Furthermore, the manipulation procedure used for the performance evaluation of BPSK can be easily extended to other signaling methods such as DPSK or FSK.

\section{Acknowledgment}

This paper was supported by the National Science Council, Taiwan, under Grants nos. NSC99-2221-E-155-033, NSC100-2220-E-155-001, and NSC100-2220-E-155-005.

\section{References}

[1] D. G. Brennan, "Linear diversity combining techniques," Proceedings of the IRE, vol. 47, no. 6, pp. 1075-1102, 1959.

[2] S. H. Lin, T. C. Lee, and M. F. Gardina, "Diversity protections for digital radio-summary of ten-year experiments and studies," IEEE Communications Magazine, vol. 6, no. 2, pp. 51-63, 1988.

[3] W. C. Jakes, Microwave Mobile Communication, IEEE Press, Piscataway, NJ, USA, 2nd edition, 1994.

[4] T. Eng, N. Kong, and L. B. Milstein, "Comparison of diversity combining techniques for Rayleigh-fading channels," IEEE Transactions on Communications, vol. 44, no. 9, pp. 11171129, 1996.

[5] G. L. Stüber, Principles of Mobile Communications, Kluwer, Norwell, Mass, USA, 1996.

[6] M. K. Simon and M.-S. Alouini, Digital Communication over Fading Channels, John Wiley - Sons, New York, NY, USA, 2000.

[7] Y.-C. Ko, M.-S. Alouini, and M. K. Simon, "Analysis and optimization of switched diversity systems," IEEE Transactions on Vehicular Technology, vol. 49, no. 5, pp. 1813-1831, 2002.

[8] W. E. Shortall, "A switched diversity receiving system for mobile radio," IEEE Transactions on Vehicular Technology, vol. 21, no. 11, pp. 1209-1275, 1973.

[9] M. A. Blanco and K. J. Zdunek, "Performance and optimization of switched diversity systems for the detection of signals with Rayleigh fading," IEEE Transactions on Communications Systems, vol. 27, no. 12, pp. 1887-1895, 1979.

[10] A. A. Abu-Dayya and N. C. Beaulieu, "Analysis of switched diversity systems on generalized-fading channels," IEEE Transactions on Communications, vol. 42, no. 11, pp. 2959-2966, 1994.

[11] C. Tellambura, A. Annamalai, and V. K. Bhargava, "Unified analysis of switched diversity systems in independent and correlated fading channels," IEEE Transactions on Communications, vol. 49, no. 11, pp. 1955-1965, 2001.

[12] H.-C. Yang and M.-S. Alouini, "Performance analysis of multibranch switched diversity systems," IEEE Transactions on Communications, vol. 51, no. 5, pp. 782-794, 2003.

[13] S. Haghani and N. C. Beaulieu, "Performance of two dualbranch postdetection switch-and-stay combining schemes in correlated rayleigh and rician fading," IEEE Transactions on Communications, vol. 55, no. 5, pp. 1007-1019, 2007.
[14] D. S. Michalopoulos and G. K. Karagiannidis, "Two-relay distributed switch and stay combining," IEEE Transactions on Communications, vol. 56, no. 11, pp. 1790-1794, 2008.

[15] V. N. Q. Bao and H. Y. Kong, "Distributed switch and stay combining for selection relay networks," IEEE Communications Letters, vol. 13, no. 12, Article ID 5353261, pp. 914-916, 2009.

[16] A. Gharanjik and K. Mohamed-Pour, "Switch-and-stay partial relay selection over Rayleigh fading channels," IET Communications, vol. 5, no. 9, pp. 1199-1203, 2011.

[17] E. A. Neasmith and N. C. Beaulieu, "New results on selection diversity," IEEE Transactions on Communications, vol. 46, no. 5, pp. 695-704, 1998.

[18] Y. G. Kim and S. W. Kim, "Optimum selection diversity for BPSK signals in Rayleigh fading channels," IEEE Transactions on Communications, vol. 49, no. 10, pp. 1715-1718, 2001.

[19] I. S. Gradshteyn and I. M. Ryzhik, Table of Integrals, Series, and Products, Academic, New York, NY, USA, 6th edition, 2000.

[20] J. G. Proakis, Digital Communications, McGraw-Hill, New York, NY, USA, 4th edition, 2001.

[21] E. Suli and D. F. Mayers, An Introduction to Numerical Analysis, Cambridge University Press, Cambridge, UK, 2003. 

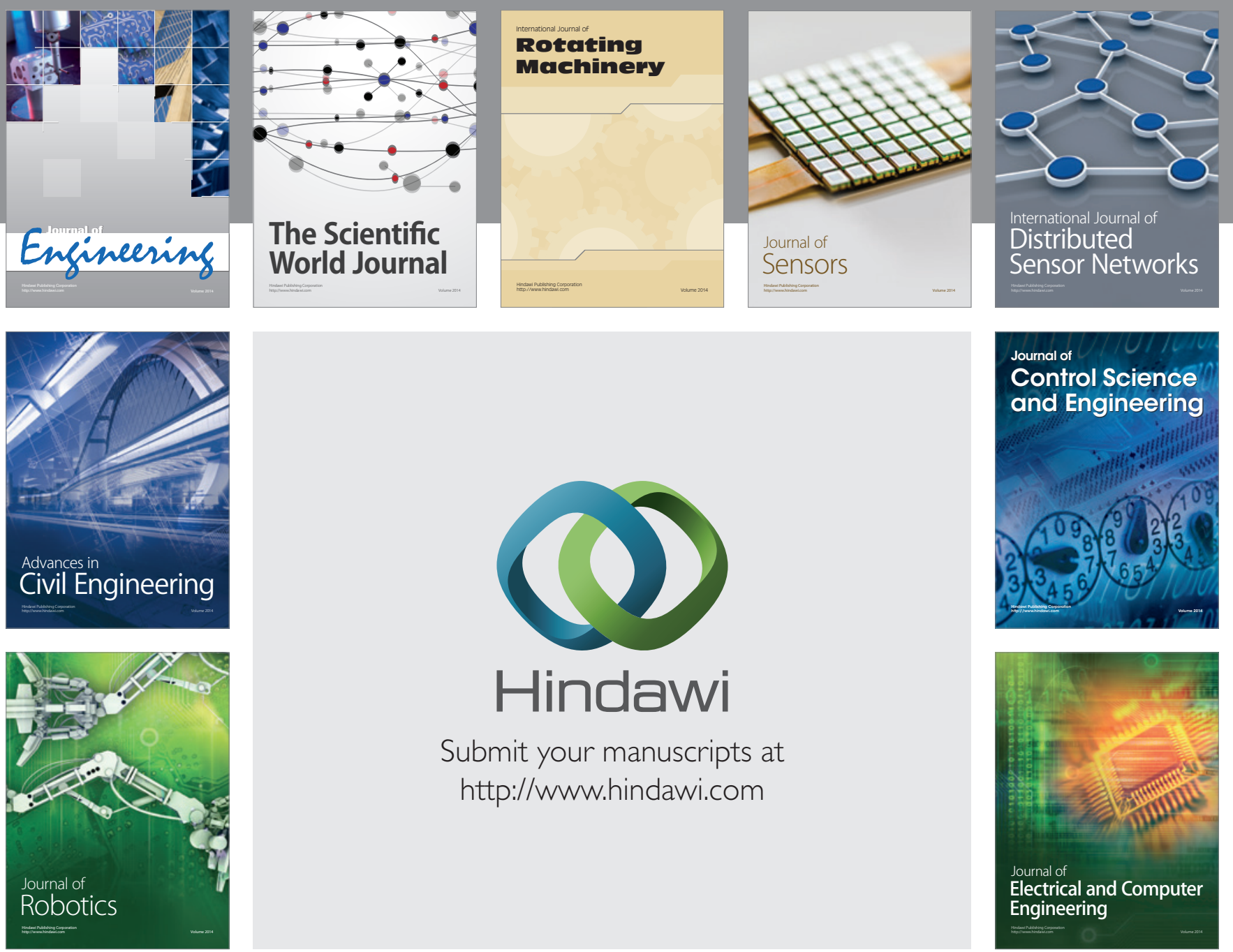

Submit your manuscripts at

http://www.hindawi.com
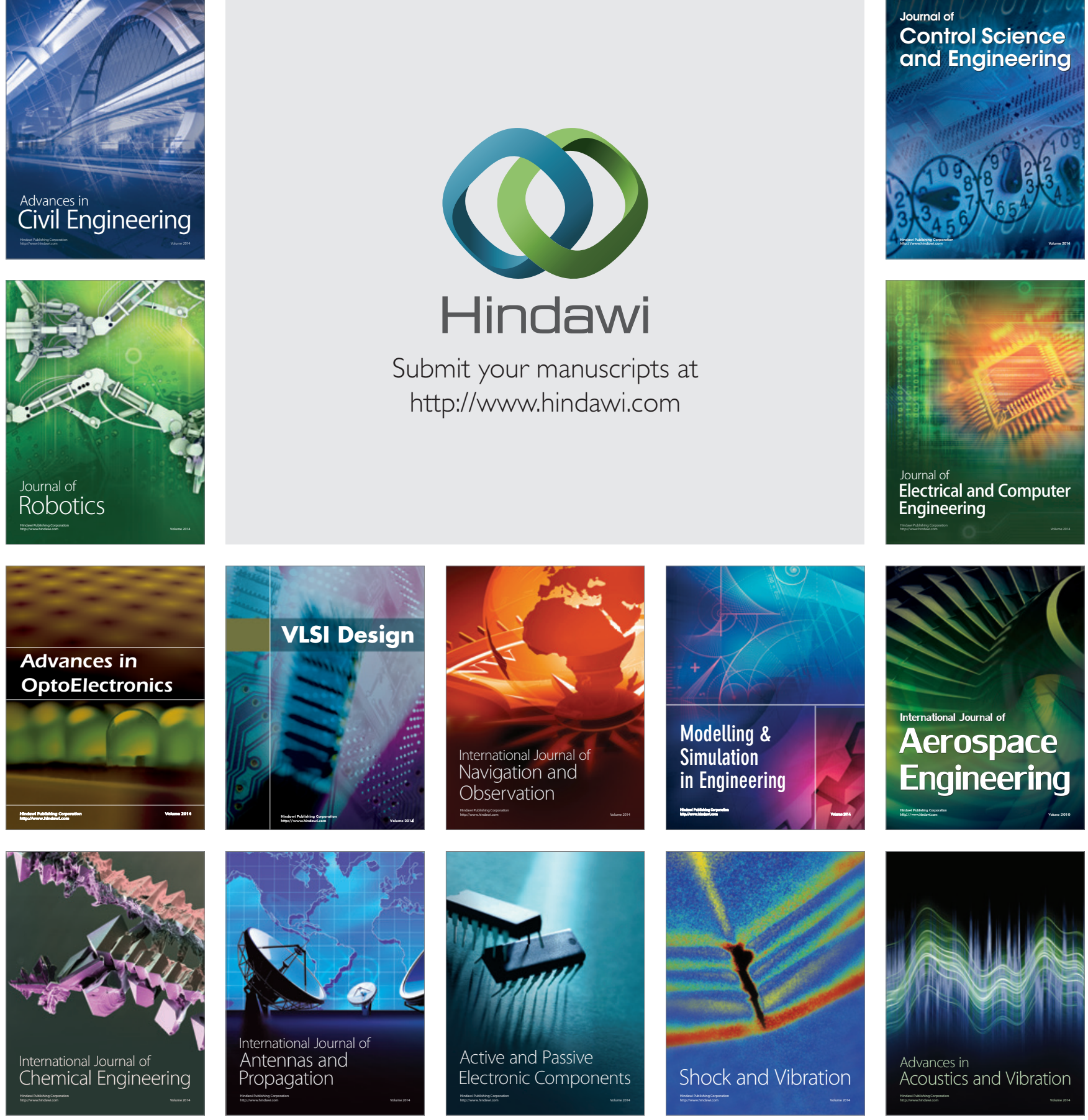